\title{
Intervenções a pacientes com mediastinite pós-esternotomia: revisão integrativa da literatura
}

\author{
Interventions for patients with post-sternotomy mediastinitis: an integrative literature review \\ Intervenciones para pacientes con mediastinitis post-esternotomía: una revisión bibliográfica \\ integradora
}

\section{RESUMO}

Objetivo: analisar as evidências disponíveis na literatura sobre as intervenções a pacientes com mediastinite pós-esternotomia. Método: Revisão integrativa com busca dos estudos primários nas bases de dados LILACS, MEDLINE (via PUBMED), CINAHL e BDENF, publicados em inglês, português e espanhol. Resultados: Os oito artigos analisados mostram que, na maioria deles, a intervenção mais utilizada foi terapia por pressão negativa, associada ou não a antibioticoterapia e cirurgia reconstrutora. Conclusão: As evidências científicas geradas fornecem subsídios para a tomada de decisão quanto às intervenções para pacientes pós-cirurgia cardíaca com mediastinite, bem como a identificação de lacunas do conhecimento, para a condução de novas pesquisas com o propósito de implementar intervenções de enfermagem a esses pacientes.

Descritores: Cirurgia Torácica; Mediastinite; Terapêutica; Literatura de Revisão.

\section{ABSTRACT}

Objective: To analyze information available in the literature on interventions for patients with mediastinitis after a cardiac surgery. Method: an integrative review of primary studies in the databases LILACS, MEDLINE (via PUBMED), CINAHL and BDENF, published in English, Portuguese, and Spanish. Results: Most of the eight analyzed articles show that the most used intervention was negative pressure therapy, whether or not associated with antibiotic therapy and reconstructive surgery. Conclusion: the scientific evidence analyzed supports decision-making processes regarding interventions for patients with mediastinitis after cardiac surgery. It also allows the identification of knowledge gaps to conduct new research and implement nursing interventions for these patients.

Descriptors: Thoracic Surgery; Mediastinitis; Therapeutics; Review.

\section{RESUMEN}

Objetivo: Analizar las evidencias disponibles en la literatura sobre intervenciones en pacientes con mediastinitis después de la cirugía cardíaca. Método: Revisión integradora con búsqueda de estudios primarios en las bases de datos LILACS, MEDLINE (vía PUBMED), CINAHL y BDENF, publicados en inglés, portugués y español. Resultados: Los ocho artículos analizados muestran que en la mayoría de ellos la intervención más utilizada fue la terapia de presión negativa, asociada o no con la terapia con antibióticos y la cirugía reconstructiva. Conclusión: La evidencia científica generada respalda la toma de decisiones con respecto a las intervenciones en pacientes después de una cirugía cardíaca con mediastinitis, así como la identificación de lagunas de conocimiento para realizar nuevas investigaciones con el propósito de implementar intervenciones de enfermería para estos pacientes.

Descriptores: Cirugía Torácica; Mediastinitis; Terapéutica; Revisión.
Carolina Letícia dos Santos $\mathrm{Cruz}^{1}$ 0000-0002-7548-2425

Ana Lúcia De Mattia² 0000-0001-7912-4943

Jaqueline Almeida Guimarães Barbosa ${ }^{2}$ $\underline{0000-0002-9175-0055}$

Fabiana Bolela ${ }^{3}$ 0000-0003-1199-6205

\section{Eline Lima Borges ${ }^{2}$ 0000-0002-0623-5308}

\section{Giovana Paula Rezende Simino ${ }^{2}$ 0000-0002-9814-3004}

\footnotetext{
${ }^{1}$ Santa Casa de Belo Horizonte.

2 Universidade Federal de Minas Gerais.

${ }^{3}$ Universidade de São Paulo.
}

Autor correspondente: Giovana Paula Rezende Simino E-mail: gsimino@yahoo.com.br 
2|Cruz CLS, Mattia AL, Barbosa JAG, et al.

\section{INTRODUÇÃO}

As doenças cardiovasculares, no Brasil e no mundo, constituem as principais causas de morte em mulheres e homens. Ademais, apresentam-se como responsáveis pela perda da força de trabalho e de renda das famílias ${ }^{(1)}$.

Para o tratamento de cardiopatias temos diversas alternativas terapêuticas disponíveis, entretanto, muitas vezes, as condutas clínicas conservadoras são insuficientes para manejo do paciente com comorbidade cardíaca e as intervenções cirúrgicas são necessárias. Dentre elas, podemos observar três tipos de cirurgia cardíaca: corretoras - fechamento de canal arterial, de septo atrial e ventricular; reconstrutoras revascularização do miocárdio, plastia de valva aórtica, mitral ou tricúspide e substitutivas - trocas valvares e transplantes. A cirurgia de revascularização do miocárdio (CRVM) é o tipo mais comum de cirurgia reconstrutora. No entanto, todas as cirurgias cardíacas são de alta complexidade ${ }^{(2)}$.

Dentre as complicações cirúrgicas temos o hematoma, seroma, infecções e deiscências. A infecção cirúrgica é caracterizada por um processo inflamatório com drenagem de secreção purulenta, tendo ou não cultura positiva, com ou sem comprometimento de tecidos adjacentes à ferida (3). Também outras variáveis contribuem para a infecção do sítio cirúrgico (ISC) na cirurgia cardíaca como, por exemplo, paciente do sexo masculino, tempo de intubação orotraqueal superior a 24 horas e reintubação no pósoperatório $^{(4)}$.

Em particular, a infecção da ferida do esterno, após cirurgia cardíaca é uma complicação muito grave. Estudo mostrou que sua incidência varia de $0,9 \%$ a $20 \%$, sendo os patógenos mais comuns, Staphylococcus coagulase negativo e Staphylococcus aureus, além de outros relacionados à infecção do esterno e mediastinite, tais como: Acinetobacter, Enterobacter cloacae, Escherichia coli e Klebsiella ${ }^{(5)}$.

Ao considerar a incidência de infecção da ferida do esterno, sua gravidade e a escassez de estudos sobre intervenções a esses pacientes, propôs-se realizar uma revisão integrativa. Frente ao exposto, considerando que a temática intervenções a pacientes com mediastinite pósesternotomia é pouco investigada, somando-se a isso a necessidade de preparo dos profissionais de saúde para o cuidado intensivo e complexo, justifica-se o interesse na condução da revisão integrativa, cujos resultados poderão gerar evidências para subsidiar o cuidado prestado. Assim, objetivou-se, analisar as evidências disponíveis na literatura, sobre as intervenções a pacientes com mediastinite pós-esternotomia.

\section{MÉTODO}

A revisão integrativa (RI) foi o método de síntese de conhecimento empregado. $\mathrm{Na}$ condução desse método, cinco etapas foram percorridas: elaboração da questão de pesquisa (identificação do problema), busca, na literatura dos estudos primários, avaliação dos estudos primários, análise dos dados e apresentação da revisão ${ }^{(6)}$.

Para nortear a condução da RI, pautada na estratégia PICOT (acrônimo de patient, exposure, comparison, outcomes e time), a seguinte questão foi formulada: Quais são as evidências disponíveis na literatura sobre o tratamento de feridas cirúrgicas para pacientes com mediastinite pósoperatória? O primeiro elemento ( $\mathrm{P}=$ paciente ou problema) consiste nos pacientes submetidos à cirurgia cardíaca com esternotomia mediana que evoluíram com deiscência e mediastinite no pósoperatório, o segundo ( $E=$ exposição) no tratamento com curativo convencional, sistema de curativo a vácuo, desbridamento cirúrgico e irrigação antisséptica, o terceiro elemento $(\mathrm{O}=$ desfechos ou resultados) no processo de cicatrização e ( $T=$ tempo) período pós-operatório.

A busca dos estudos primários foi realizada, por meio de busca online em quatro bases de dados da área da saúde: Literatura Latino Americana e do Caribe em Ciências da Saúde (LILACS), Medical Literature Analysis and Retrieval System Online (MEDLINE via PUBMED), Cumulative Index to Nursing and Allied Health Literature (CINAHL) e Banco de Dados em Enfermagem (BDENF).

Os descritores e as palavras-chave foram estabelecidos de acordo com cada base de dados selecionada, para garantir busca rigorosa e ampla dos estudos primários sobre o tópico de interesse, associados ao acrônimo PICOT delimitado para a estratégia de busca (Figura 1). Para a busca das publicações em cada base de dados, os descritores e as palavras-chave foram cruzados entre si, por meio de operações táticas, utilizando-se os operadores booleanos AND e OR. 
Figura 1 - Descritores e palavras-chave selecionadas em cada base de acordo com o acrômio PICOT.

\begin{tabular}{|c|c|c|c|}
\hline \multicolumn{4}{|c|}{ Descritores e palavras chaves } \\
\hline LILACS & MEDLINE & CINAHL & BDENF \\
\hline $\begin{array}{l}\text { Cirurgia cardíaca } \\
\text { Cirurgia do coração } \\
\text { Cirurgia torácica } \\
\text { Mediastinite } \\
\text { Mediastinite-complicações } \\
\text { Deiscência } \\
\text { Infecção } \\
\text { Curativo } \\
\text { Curativo-reabilitador } \\
\text { Curativo-recuperativo Curativos de } \\
\text { hidrogel } \\
\text { Terapêutica } \\
\text { Tratamento } \\
\text { Ação terapêutica } \\
\text { Medida terapêutica } \\
\text { Procedimentos terapêuticos }\end{array}$ & $\begin{array}{l}\text { Thoracic surgery/complications } \\
\text { Surgery, Thoracic } \\
\text { Surgery } \\
\text { Cardiac Surgery } \\
\text { Heart Surgery } \\
\text { Cardiac Surgery } \\
\text { Bandage } \\
\text { Mediastinitis }\end{array}$ & $\begin{array}{l}\text { Cardiac surgery } \\
\text { Mediastinitis }\end{array}$ & $\begin{array}{l}\text { Cirurgia cardíaca } \\
\text { Mediastinite }\end{array}$ \\
\hline
\end{tabular}

Fonte: Dos autores.

As estratégias de busca foram construídas por uma bibliotecária e pelas pesquisadoras com o intuito de tornar a busca mais sensível. Dessa forma, as estratégias foram diferentes para cada base de dados, utilizando-se descritores controlados e não controlados presentes no vocabulário de Descritores em Ciências da Saúde (DECS) da Biblioteca Virtual em Saúde. A estratégia para a base de dados LILACS foi ("CIRURGIA CARDIACA") or "CIRURGIA DO CORACAO") or "CIRURGIA TORACICA" [Palavras] and ( ("MEDIASTINITE" or "MEDIASTINITECOMPLICACOES") or "DEISCENCIA") or "INFECCAO") or "DEISCENCIA" [Palavras] and ( ( ( ) ("CURATIVO" or "CURATIVO-REABILITADOR" or "CURATIVO-RECUPERATIVO" or "CURATIVOS DE HIDROGEIS") or "TERAPEUTICA") or "TRATAMENTO") or "ACAO TERAPEUTICA") or "MEDIDA TERAPEUTICA") or "PROCEDIMENTOS TERAPEUTICOS" [Palavras] que recuperaram 47 estudos. A estratégia para a MEDLINE foi /((/(("thoracic surgery/complications" or Surgery, Thoracic or Surgery or Cardiac Surgery or Heart Heart Surgery or Cardiac Surgery)))) AND Bandage AND Mediastinitis, obtendo-se o total de 45 estudos. A estratégia para as bases de dados BDENF e CINAHL foi Cirurgia Cardíaca e Mediastinite e seu respectivo descritor em inglês (Cardiac surgery and Mediastinites) que recuperou 3 e 59 estudos, respectivamente.

Para a seleção dos estudos desta revisão, os seguintes critérios de inclusão foram adotados: estudos com desenho metodológico primário que investigaram as intervenções para pacientes com mediastinite pós-esternotomia; pesquisas publicadas em inglês, português e espanhol e nos últimos 10 anos. A delimitação do período foi estabelecida para assegurar quantitativo adequado de pesquisas, sendo que um número elevado de estudos primários poderia inviabilizar a condução da revisão integrativa ou introduzir vieses nas etapas seguintes do método. Critérios de exclusão: estudos com crianças.

A partir dos resultados das estratégias de busca empregadas nas bases de dados selecionadas para a condução da revisão, um arquivo foi importado para o gerenciador de referências EndNote, versão X5. Para o gerenciamento das referências, foram criadas pastas para cada base de dados e realizadas filtragens para os estudos duplicados, livros, dissertações, teses e outros textos não científicos, em idiomas não delimitados e com o período de publicação fora do estabelecido. A pré-seleção dos estudos primários por meio da leitura dos títulos e resumos foi realizada e, finalmente, feita a seleção final das pesquisas para leitura na íntegra.

$\mathrm{Na}$ Figura 2, apresenta-se o fluxograma de seleção dos estudos primários. Um instrumento elaborado por pesquisador nacional foi utilizado para extração de dados dos estudos primários, o qual contém conjunto de itens que possibilitam a identificação das pesquisas, características metodológicas, principais resultados e conclusões $^{(7)}$. 
4|Cruz CLS, Mattia AL, Barbosa JAG, et al.

Figura 2 - Fluxograma de seleção dos estudos primários.

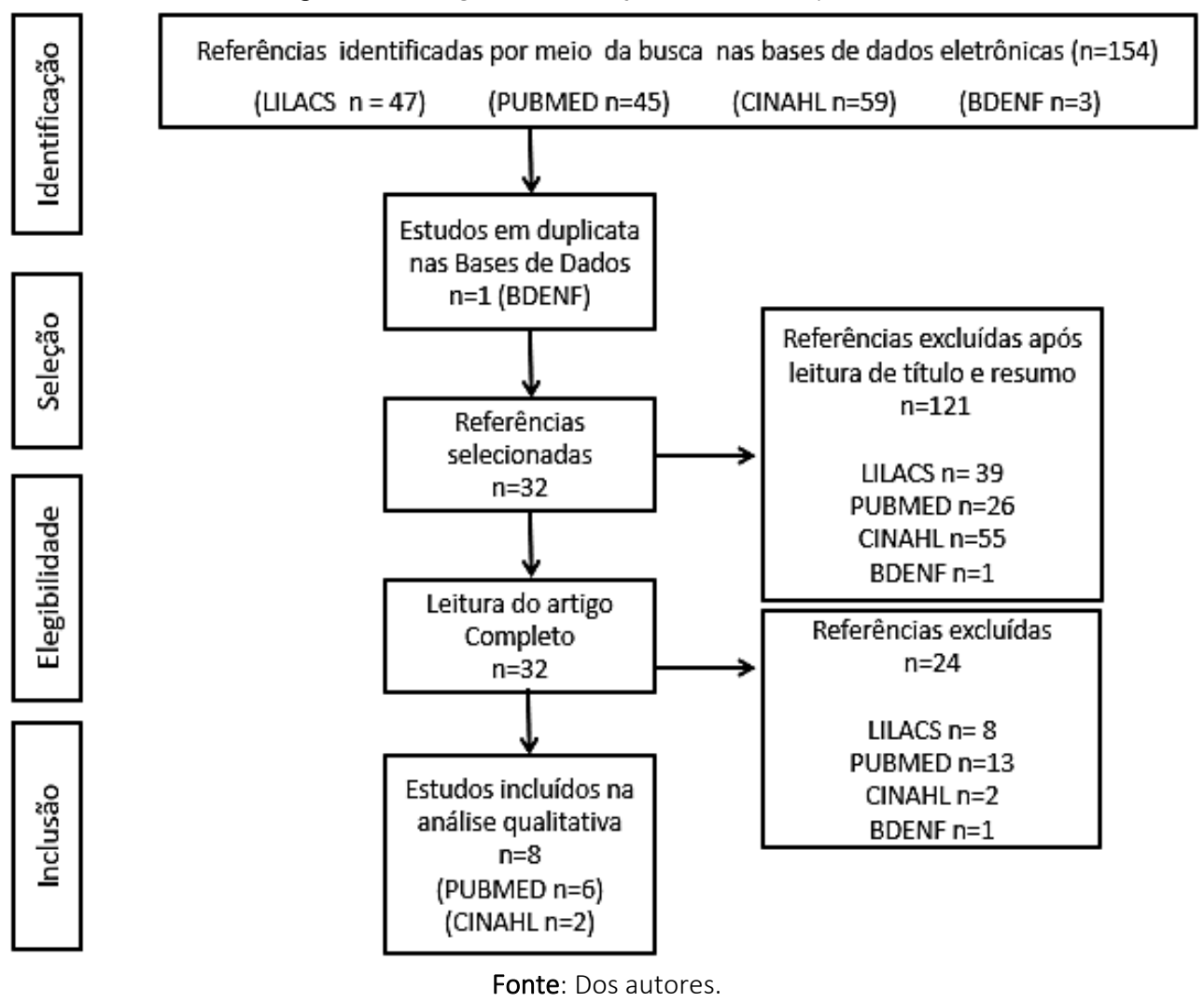

A classificação de hierarquias de evidências de acordo com o tipo de questão clínica dos estudos foi empregada. A questão clínica pode ser: (a) de significado (com cinco níveis de evidência, sendo a mais forte, ou seja, nível I, evidências obtidas de metassíntese de estudos qualitativos, e a menor, nível $V$, evidências de opinião de especialistas), (b) de prognóstico, predição ou etiologia (com cinco níveis de evidência, sendo a mais forte, ou seja, nível I, evidências obtidas de síntese de estudos de coorte ou caso-controle, e a menor, nível $V$, evidências de opinião de especialistas) e (c) de intervenção, tratamento ou diagnóstico/teste diagnóstico (com sete níveis de evidência, sendo a mais forte, ou seja, nível I, evidências obtidas de revisão sistemática ou metanálise, e a menor, nível VII, evidências de opinião de especialistas) ${ }^{(6)}$. A síntese dos resultados da revisão foi realizada na forma descritiva. Assim, os oito estudos primários foram incluídos na amostra da revisão.

Os estudos que atenderam aos critérios de inclusão foram, em sua maioria, realizados em países europeus, sendo que todos foram publicados em língua inglesa. Vale destacar, no entanto, que um dos estudos foi publicado em periódico asiático. O período de publicação dos estudos inseridos, nesta revisão, foi de 2003 a 2014.

\section{RESULTADOS E DISCUSSÃO}

Foi possível identificar estudos com curto e longo período de recrutamento de pacientes, o que trouxe amostras com tamanhos bem variados e diferentes tipos de intervenções. Em relação à base de dados, oito estudos primários foram identificados.

A maioria dos estudos tinha mais de cinco autores, sendo que o número máximo de autores foi oito. Quatro estudos trouxeram a frequência das intervenções, segundo o sexo dos pacientes. Nessas amostras, foi possível identificar que o sexo masculino foi mais frequente.

Algumas variáveis explicativas foram coletadas dos estudos e descritas no Quadro 1. Destaca-se que alguns estudos não descrevem características dos participantes quanto ao tipo de procedimento cirúrgico realizado, assim como a presença de comorbidade dos seus pacientes. 0 procedimento cirúrgico mais frequente foi a revascularização do miocárdio e a comorbidade foi o DM. Diferentes tipos de intervenções foram propostos aos pacientes para controle da mediastinite, sendo que a utilização do sistema de fechamento por vácuo foi o mais utilizado para a avaliação de eficácia de tratamento comparado a outras modalidades convencionais de tratamento. 
Quadro 1 - Caracterização dos estudos, segundo os objetivos, intervenções e nível de evidência. Belo Horizonte, 2017.

\begin{tabular}{|c|c|c|c|}
\hline Autor (es) & Objetivo & Intervenções & NE* \\
\hline 1. Simek, M. et al.(8) & $\begin{array}{l}\text { Verificar se o curativo a vácuo é um } \\
\text { sistema seguro e uma opção } \\
\text { confiável no tratamento de feridas } \\
\text { infectadas de esterno extensas em } \\
\text { cirurgias cardíacas. }\end{array}$ & $\begin{array}{l}\text { O curativo a vácuo é uma opção segura e confiável no } \\
\text { tratamento da infecção da ferida esternal em cirurgia } \\
\text { cardíaca. É considerado um complemento efetivo no } \\
\text { tratamento convencional para o tratamento de } \\
\text { infecções extensas e, potencialmente, fatais após } \\
\text { cirurgia cardíaca, particularmente, na presença de } \\
\text { fatores de risco. }\end{array}$ & IV \\
\hline 2. Grauhan, O. et al.(9) & $\begin{array}{l}\text { Verificar se o manejo de incisão } \\
\text { cirúrgica } \\
\text { utilizando a pressão negativa sobre } \\
\text { as incisões cirúrgicas fechadas por } \\
\text { seis a sete dias } \\
\text { reduz as infeç̧ões de feridas em } \\
\text { uma população de pacientes com } \\
\text { esternotomia total. }\end{array}$ & $\begin{array}{l}\text { A aplicação do manejo da incisão cirúrgica em incisões } \\
\text { limpas e fechadas nos primeiros seis a sete dias de pós- } \\
\text { operatório reduziu a probabilidade de infecção pós- } \\
\text { operatória da ferida após esternotomia mediana não } \\
\text { somente em pacientes de alto risco, mas também em } \\
\text { um paciente abrangente população. }\end{array}$ & IIB \\
\hline $\begin{array}{l}\text { 3. Vos, Roemer J. et } \\
\text { al.(10) }\end{array}$ & $\begin{array}{l}\text { Analisar os desfechos clínicos do } \\
\text { curativo a vácuo em comparação ao } \\
\text { tamponamento aberto e estimar os } \\
\text { fatores de risco para mortalidade. }\end{array}$ & $\begin{array}{l}\text { A terapia com curativo a vácuo é superior ao curativo } \\
\text { aberto, e resulta em menor tempo de permanência em } \\
\text { terapia intensiva e melhora da sobrevivência. }\end{array}$ & IV \\
\hline 4. Feo, M. et al.(11) & $\begin{array}{l}\text { Avaliar a experiência de } 30 \text { anos no } \\
\text { tratamento da infecção profunda da } \\
\text { ferida esternal após cirurgia } \\
\text { cardíaca. }\end{array}$ & $\begin{array}{l}\text { O desbridamento cirúrgico precoce e a aplicação de } \\
\text { curativo a vácuo, seguido de reconstrução por cirurgia } \\
\text { plástica proporcionou uma taxa satisfatória de cura e } \\
\text { uma boa taxa de sobrevivência. }\end{array}$ & IIC \\
\hline 5. Zeitani, J. et al.(12) & $\begin{array}{l}\text { Comparar o tratamento } \\
\text { convencional com o desbridamento } \\
\text { cirúrgico e fechamento direto. }\end{array}$ & $\begin{array}{l}\text { A esternotomia é um tratamento seguro e válido. A } \\
\text { ferida infectada não é uma contraindicação para } \\
\text { tratamento cirúrgico. } \\
\text { O fechamento primário pode contribuir para reduzir o } \\
\text { risco de Infecção posterior e contribui para a redução do } \\
\text { tempo de cicatrização, desconforto dos pacientes, } \\
\text { redução dos custos hospitalares e carga de trabalho da } \\
\text { equipe de saúde. }\end{array}$ & IIC \\
\hline $\begin{array}{l}\text { 6. Domkowski, P.W. et } \\
\text { al.(13) }\end{array}$ & $\begin{array}{l}\text { Verificar a eficácia do sistema de } \\
\text { curativo a vácuo como adjuvante da } \\
\text { terapia convencional ou como } \\
\text { terapia única para pacientes com } \\
\text { doença cardíaca mediastinite } \\
\text { cirúrgica. }\end{array}$ & $\begin{array}{l}\text { A drenagem assistida por vácuo é uma terapia eficaz } \\
\text { para a mediastinite, após debridamento ou antes da } \\
\text { colocação de um retalho de tecido vascularizado. }\end{array}$ & IV \\
\hline 7. Bapat, $V$ et al.(14) & $\begin{array}{l}\text { Verificar o uso da terapia de } \\
\text { fechamento assistida por vácuo, no } \\
\text { tratamento de infecção da ferida } \\
\text { pós-esternotomia com ênfase nos } \\
\text { problemas recorrentes relacionados } \\
\text { à ferida após o uso de curativo a } \\
\text { vácuo e seu tratamento. }\end{array}$ & $\begin{array}{l}\text { A terapia a vácuo é segura e confiável opção no } \\
\text { tratamento da infecção da ferida esternal. No entanto, } \\
\text { o uso prolongado do sistema a vácuo como substituto } \\
\text { para o fechamento cirúrgico da ferida esternal parece } \\
\text { estar associado a problemas recorrentes da ferida no } \\
\text { esterno. A estratégia de uso de curativo a vácuo por um } \\
\text { curto período seguido de fechamento cirúrgico precoce } \\
\text { parece favorável. }\end{array}$ & IV \\
\hline $\begin{array}{l}\text { 8. Karaca, S; } \\
\text { Kalangos }{ }^{(15)}\end{array}$ & $\begin{array}{l}\text { Descrever o tratamento de M. } \\
\text { hominis mediastinite, após cirurgia } \\
\text { cardíaca com aspiração assistida a } \\
\text { vácuo, combinada com a terapia } \\
\text { com irrigação antisséptica, diluída } \\
\text { para a erradicação bacteriana. }\end{array}$ & $\begin{array}{l}\text { A utilização adequada de agentes bactericidas no tecido } \\
\text { infectado pode ser difícil, especialmente em } \\
\text { mediastinite com Mycoplasma espécies. A irrigação } \\
\text { contínua fechada tem a vantagem de lavagem de } \\
\text { bactérias, tecido necrótico, fibrina e coágulos. A } \\
\text { erradicação eficaz de M. hominis é associada à terapia } \\
\text { intravenosa com antibióticos e prepara o leito da ferida. }\end{array}$ & IV \\
\hline
\end{tabular}

*NE: nível de evidência

Fonte: Dos autores.

Esta revisão permitiu identificar e analisar intervenções realizadas para pacientes com complicações graves no pós-operatório de esternotomia, após cirurgias cardíacas.

As mediastinite pós-operatórias foram definidas, nestes estudos, considerando-se classificações estabelecidas pelo Centro de
Controle de Doenças Norte-Americano, (Central Disease Control - CDC). A mediastinite foi relatada como uma complicação rara, porém, que se apresenta como um fator de risco para aumento do tempo de morbidade, tempo de internação, aumento dos custos e também para o aumento mortalidade pós-operatória(8-15). 
A mediastinite pós- esternotomia mediana, também chamada de infecção da ferida esternal, é uma complicação grave, para o fechamento do diagnóstico e deverá seguir um dos seguintes critérios: um organismo isolado de cultura de tecido mediastinal ou fluido, evidência de mediastinite observada durante a operação, sinais de infecção como dor torácica esternal, instabilidade ou febre, associado à saída de exsudato purulento do mediastino ou da ferida cirúrgica ${ }^{(8-15)}$.

Apenas um estudo subdividiu as feridas de mediastinite, utilizando as classificações de gravidade do tipo I ao tipo V. Neste estudo, houve maior frequência dos subtipos II e III ${ }^{(13)}$.

Sua patogenia é extremamente complexa e está associada a questões multifatoriais. A literatura revisada aponta como preditivos de risco os seguintes fatores: doença cardiovascular, obesidade, DM, doença pulmonar obstrutiva crônica e insuficiência cardíaca, sendo que presença de um desses diagnósticos, ou fatores de risco, aumentam o risco de o paciente evoluir com mediastinite $^{(8-15)}$.

Os estudos desta revisão integrativa trouxeram, em sua maioria $n=7(87,5 \%)$, a terapia com sistema de fechamento por vácuo, também conhecido na literatura por terapia por pressão negativa (TPN) ${ }^{(8-11,13-15)}$.

Dessa maneira, destacaremos a utilização do VAC, nesta discussão. Para tal, trouxemos a discussão em duas categorias: tratamento por VAC (subdivididos em 4 itens) e tratamento cirúrgico exclusivo:

\section{1) Tratamento por VAC}

\section{Indicação e utilização do sistema de fechamento assistido por vácuo (VAC)}

Os estudos utilizaram o VAC para feridas esternais infectadas superficiais e profundas póscirurgia cardíaca $^{(8)}$. Outros estudos não descreveram as características da profundidade das lesões esternais ${ }^{(9,11-13)}$. O estudo 8 caracterizou a ferida esternal como profunda.

Os pacientes receberam antibioticoterapia, de acordo com os microorganismos isolados e seu uso foi descontinuado, a partir da ausência de sinais e sintomas de infecção, cultura negativa e formação de tecido de granulação ${ }^{(8)}$.

A terapia de VAC era descontinuada quando havia decréscimo dos níveis de proteína $C$ reativa menor que $50 \mathrm{mg} / \mathrm{l}$. Para pacientes com feridas profundas 0 procedimento cirúrgico para fechamento era realizado o mais precocemente possível ${ }^{(8,10)}$. Em outro estudo, além desses critérios, o paciente era descontinuado quando a febre cessava ${ }^{(11)}$. Ainda, mais um estudo citou que o tempo de aplicação de vácuo foi de 6-7dias sem explicitação de outros critérios ${ }^{(9)}$.

A pressão negativa foi inferior a $125 \mathrm{mmHg}$ $(8,9,11)$. Ainda, em um dos estudos foi descrito que a pressão utilizada foi de 75 a 125 mmHg e o sistema de vácuo era trocado duas vezes na semana. Nos demais, não há descrição da pressão utilizada ${ }^{(10)}$.

\section{Intervenções pré-utilização do VAC}

Alguns estudos relataram intervenções realizadas previamente à administração da terapia por VAC.

Dentre os cuidados para administração do VAC, destaca-se que, para pacientes com exposição profunda foi vedada qualquer possibilidade de contato com o coração(8,11,15). No estudo 4, a proteção foi de silicone. O procedimento de desbridamento do tecido necrótico e intensa irrigação com solução salina fisiológica precedeu a utilização do vácuo ${ }^{(8)}$.

Ferida foi coberta com filme de poliuretano transparente com espessura entre 400 a $600 \mu \mathrm{m}$ foi descrita em um dos estudos ${ }^{(8)}$.

A técnica de utilização do $V^{\circ} C^{\circ}$ envolveu a montagem de uma peça cortada de espuma de poliuretano no esterno ${ }^{(11)}$.

A linha de instilação foi para o antisséptico fluido Polihexanid B $2 \%$ onde foi distribuído otimamente sobre o leito da ferida em 20 segundos. A tubulação distal estava conectada para a embalagem VAC. O líquido antisséptico permaneceu na cavidade do mediastino por 10 minutos $^{(11)}$.

\section{Eficácia da Terapia por VAC e seus desdobramentos}

Os desfechos da utilização do VAC foram medidos em diferentes perspectivas nos estudos. Destaca-se o tempo de internação, mortalidade e tempo de fechamento das feridas.

Em um dos estudos, todos os pacientes foram submetidos à terapia por vácuo, ou seja, não houve grupo controle. O tempo médio para resolução da infecção foi de 9,2 dias (intervalo de 6 a 21 dias $)^{(8)}$

Os pacientes submetidos à tratamento com VAC (Prevena ${ }^{\circledR}$ Therapy) obtiveram resposta favorável para o controle da mediastinite com diferença, estatisticamente significativa, quando 
comparados com os pacientes tratados com cobertura convencional. Ressalta-se que não foi descrito o que se considerou como cobertura convencional. $(p<0,05 \text {; Odds ratio } 2.74)^{(9)}$.

A mortalidade foi menor em pacientes submetidos compressas de gazes (procedimento realizado diariamente) foi comparada com terapia por vácuo. A mortalidade hospitalar foi estatisticamente significativa menor nos pacientes que foram submetidos à terapia por vácuo $(p=0,0032)$. Esses dados foram coletados por período de 10 anos, entre os anos 2000 e $2010^{(10)}$.

O uso da terapia por pressão negativa, conhecida, internacionalmente, pela sigla VAC (Vacuum Assisted Closure $^{\circledR}$ ) em um centro de referência em cirurgia cardíaca, por três décadas, mostrou se mais eficaz do que outras terapias. Nesse período, 22.366 pacientes foram submetidos à cirurgia cardíaca por esternotomia completa. A taxa de infecção foi de 0,89\% (200 pacientes). Dentre esses pacientes com infecção do sítio cirúrgico, com progressão para mediastinite, a permanência média de internação hospitalar foi, estatisticamente significativa, maior no grupo de pacientes tratados com antibioticoterapia (grupo 1) e também naqueles submetidos a desbridamento cirúrgico (se falha irrigação por 10 dias e cobertura com açúcar e tratamento com hiperbárica) (grupo 2) com àqueles submetidos a desbridamento e VAC e reconstrução do músculo peitoral. Além disso, o tempo para cessar a febre foi menor no grupo 3, quando comparado ao grupo 2 e 1 . Além disso, a contagem de proteína $C$ reativa, em 7 dias e a contagem de leucócitos também em 7 dias, foi menor, estatisticamente, significativo no grupo 3. Mortalidade hospitalar no grupo 1 foi maior, com diferença estatisticamente significativa quando comparada com os grupos 2 e 3. Não houve diferença estatística entre os grupos 2 e 3 para mortalidade. (estudo 4) ${ }^{(11)}$.

O vácuo associado ao desbridamento e associado ao procedimento cirúrgico com reconstrução muscular (omental ou peitoral) mostrou-se benéfico para reduzir a infecção. (estudo 6) ${ }^{(14)}$.

O relato da antibioticoterapia foi descrita em relato de caso para paciente submetido à cirurgia de substituição de aorta. Foi utilizado Imipenem e vancomicina de maneira empírica. O paciente, posteriormente, foi diagnosticado com M.hominis. e foi realizado troca de antibioticoterapia e utilização de VAC-Instill ${ }^{\circledR}$ (instilação de solução Prontosan, Polihexanid 0,02\%)(15).
A terapia por vácuo, quando comparada à terapia com cobertura convencional, irrigação da ferida esternal com peróxido de hdrogênio, solução salina fisiológica e solução de povidineiodo e coberta com prata se mostrou mais efetiva.

\section{Eventos Adversos}

O relato dos eventos adversos oriundos da terapia por pressão negativa foi de pouco descrito nos estudos.

A pressão contínua com valor maior que 125 $\mathrm{mmHg}$ causou desconforto, no entanto, não foi encontrada nenhuma complicação relacionada ao uso do $V_{A C}{ }^{(8)}$.

A VAC foi bem tolerada em todos os pacientes ${ }^{(9)}$. O uso prolongado da terapia VAC (período maior que 21 dias) trouxe indícios de provocar necrose óssea e infecção óssea, sendo necessário desbridamento do tecido(14).

\section{2) Tratamento Cirúrgico}

No período de 1999 a 2002, 2400 pacientes foram submetidos a esternotomia mediana. Destes, $0.125 \%(n=3)$ tiveram mediastinite e 2,75\% $(n=66)$ tiveram deiscência profunda e 5,75\% ( $n=138)$ tiveram deiscência superficial ${ }^{(12)}$.

Todos os pacientes submetidos à cirurgia de esternotomia receberam antibiótico profilaxia com $2 \mathrm{~g}$ de ceftizoxime e antissepsia da pele com povidine iodo ${ }^{(12)}$

Houve uma diferença impressionante no período de tratamento, como o número de medicamentos necessários, entre os dois métodos, com o método cirúrgico, claramente, provando ser mais eficaz. O fechamento primário direto da deiscência da ferida superficial, após a esternotomia mediana parece ser um tratamento seguro e efetivo ${ }^{(12)}$.

Uma cultura positiva de feridas abertas não parece contraindicação para o fechamento primário e pode reduzir o risco de propagação da infecção. Quando comparado com a cura secundária, o tempo de cura, o desconforto dos pacientes, os custos hospitalares e o envolvimento da equipe hospitalar são, significativamente, reduzidos. O período de tratamento foi de 29,7 dias (intervalo de 2 a 144 dias) para o grupo 1 (desbridamento) e 12,2 dias (intervalo de 2 a 37 dias), incluindo o dia do fechamento cirúrgico direto e remoção de suturas, para o grupo 2 (tratamento cirúrgico com sutura completa) ( $p=$ 0,0001). O número médio de tratamentos médicos 
foi de 9,4 por paciente no grupo 1 e 3,7 por paciente no grupo $2(p=0,0001)^{(12)}$.

A literatura descreve que a terapia de pressão negativa é um tipo de tratamento ativo da ferida que promove sua cicatrização, uma vez que mantém o ambiente úmido, por meio de uma pressão subatmosférica controlada e aplicada localmente ${ }^{(16)}$.

No cenário brasileiro, atualmente, a intervenção mais utilizada para o manejo da ferida esternal pós- cirurgia cardíaca é a utilização de coberturas convencionais que mantêm o tecido úmido para propiciar granulação do tecido. No entanto, estudos tem relatado a utilização da terapia por pressão negativa (TPN) também conhecida como sistema de fechamento por vácuo (VAC) ou terapia de pressão negativa ${ }^{(17)}$.

Um estudo brasileiro comparou a eficácia desse sistema internacional com um patenteado na Universidade de São Paulo (USP). O sistema nacional utiliza produtos já conhecidos no mercado (compressa de gaze dobrada, esponja estéril retirada da escova de degermação de mãos utilizada pré-procedimento cirúrgico, cateter uretral e sistema de vácuo com regulador de pressão e coletor descartável). O pesquisador não encontrou diferença, estatisticamente significativa, de eficácia entre o sistema internacional e o patenteado no Brasil, o que demonstra que o produto brasileiro pode ser utilizado. No entanto, custo com o produto brasileiro por paciente é muito inferior $R \$ 47,89$ comparado ao norte-americano $\mathrm{R} \$ 2.757,40^{(18)}$.

\section{CONCLUSÃO}

A utilização do sistema de fechamento, por terapia de pressão negativa, apresentou maior efetividade do que coberturas convencionais com trocas diárias e irrigação com soluções diversas (peróxido de hidrogênio, povidine iodo e solução salina). Outra evidência é o tratamento cirúrgico para fechamento da deiscência, ser mais efetivo do que o fechamento da ferida esternal, por segunda intenção.

Os pacientes possuem algumas comorbidades presentes, como DM, doença renal crônica, hipertensão arterial sistêmica e são, em sua maioria, do sexo masculino e com idade superior a 50 anos. No entanto, essas variáveis não se mostraram estatisticamente significativas para o aparecimento da mediastinite, nestes estudos.

Quanto às evidências para a prática clínica, ainda falta embasamento para estabelecer protocolos seguros, uma vez que o desenho dos estudos carece de rigor metodológico, com poucas informações acerca dos métodos estatísticos e alocação dos pacientes nos grupos de intervenções.

Destaca-se pela avaliação das pesquisas, que a maioria dos estudos apresenta desenhos metodológicos considerados fracos, para recomendação de intervenções, que podem estar presentes na Revisão Integrativa de Literatura. Outra limitação da Revisão Integrativa é que os descritores escolhidos recuperam nas bases de dados uma amostra da publicação cientifica, o que pode não retratar a completude das evidências.

Nesta revisão, contribuiu-se para sintetizar e atualizar o conhecimento existente para o tratamento de mediastinite, em pacientes submetidos à esternotomias para cirurgias cardíacas. Salienta-se que o paciente necessitará de Enfermagem qualificada para o seu atendimento, pois se encontram com complicação pós-operatória grave e, portanto, esses resultados poderão subsidiar os cuidados de enfermagem baseados em evidência científica, demonstrando as técnicas utilizadas e a sua eficácia.

\section{REFERÊNCIAS}

1 - Malta DC, Teixeira R, Oliveira GMM, Ribeiro ALP. Mortalidade por doenças cardiovasculares segundo o sistema de informação sobre mortalidade e as estimativas do estudo carga global de doenças no Brasil, 2000-2017. Arq Bras Cardiol. 2020;115(2):152-60. DOI:

\subsection{0/abc. 20190867}

2 - Amorim TV, Salimena AMO. Processo cirúrgico cardíaco e suas implicações no cuidado de enfermagem: Revisão/reflexão. HU Rev. 2015 [citado em 15 mar 2020]; 41(3-4):149-54. Disponível em: https://periodicos.ufjf.br/index.php/hurevista/arti cle/view/2171

3 - Oliveira MC, Andrade AYT, Turrini RNT, Poveda VB. Negative pressure wound therapy in the treatment of surgical site infection in cardiac surgery. Rev Bras Enferm. 2020;73(5):1-8. DOI: $\underline{10.1590 / 0034-7167-2019-0331}$

4 - Brasil. Agência de Vigilância Sanitária. Medidas de prevenção de infecção relacionada a assistência a saúde. 2a ed. Brasília: Anvisa; 2017. 
5 - Assunção TP, Pontes BCD, Damasceno CAV. Prevalência de infecções em suturas de cirurgias de revascularização do miocárdio. Rev Bras Cir Cardiovasc. 2011;26(1):43-6. DOI: 10.1590/S010276382011000100010

6 - Mendes KS, Silveira RCCP, Galvão CM. Revisão integrativa: Método de pesquisa para a incorporação de evidências na saúde e na enfermagem. Texto Contexto-Enferm. 2008;17(4):758-64. DOI: 10.1590/S0104$\underline{07072008000400018}$

7 -Santos CC, Pimenta CAM, Nobre MRC. A estratégia PICO para a construção da pergunta de pesquisa e busca de evidências. Rev Latino-Am Enfermagem 2007;15(3):508-11. DOI: $\underline{10.1590 / 50104-11692007000300023}$

8-Simek M, Nemec P, Zalesak B, Kalab M, Jecminkova L, Hajec R, et al. Vacuum-assisted closure in the treatment of sternal wound Infection after cardiac surgery. Biomed Pap Med Fac Univ Palacky Olomouc Czech Repub. 2007;151(2):295-9. DOI: $10.5507 /$ bp. 2007.050

9 - Grauhan O, Navasardyan A, Tutkun B, Hennig F, Müller $P$, Hummel $M$, et al. Effect of surgical incision management on wound infections in a poststernotomy patient population. Int Wound J. 2014;11(1):6-9. DOI: 10.1111/iwj.12294

10 - Vos RJ, Yilmaz A, Sonker U, Kelder JC, Kloppenburg JTL. Vacuum-assisted closure of poststernotomy mediastinitis as compared to open packing. Interact Cardiovasc Thorac Surg. 2012;14(1):17-21. DOI: 10.1093/icvts/ivr049

11 - Feo M, Vicchio M, Santè P, Cerasuolo F, Nappi G. Evolution in the treatment of mediastinitis: Single-center experience. Asian Cardiovasc Thoracic Ann. 2011;19(1):39-43. DOI: $\underline{10.1177 / 0218492310395789}$

12 - Zeitani J, Bertoldo F, Bassano C, Peppo AP, Pellegrino A, Fakhri FM, et al. Superficial wound dehiscence after median sternotomy: Surgical treatment versus secondary wound healing. Ann Thorac Surg. 2004;77(2):672-5. DOI: $\underline{10.1016 / 50003-4975(03) 01594-7}$

13 - Domkowski PW, Smith ML, Gonyon Júnior DL, Drye C, Wooten MK, Levin LS, et al. Evaluation of vacuum-assisted closure in the treatment of poststernotomy mediastinitis. J Thorac Cardiovasc
Surg. 2003;126(2):386-9. DOI: 10.1016/s0022$\underline{5223(03) 00352-0}$

14 - Bapat V, El-Muttardi N, Young C, Venn G, Roxburgh J. Experience with vacuum-assisted closure of sternal wound infections following cardiac surgery and evaluation of chronic complications associated with its use. J Card Surg. 2008;23(3):227-33. DOI: $\quad$ 10.1111/i.15408191.2008.00595.x

15 - Karaca S, Kalangos A. Vacuum-assistedclosure (VAC)-Instill ${ }^{\circledR}$ with continuous irrigation for the treatment of Mycoplasma hominis mediastinitis [relato de caso]. Int Wound J. 2015;12(5):595-7. DOI: 10.1111/iwj.12234

16 - Lima RVKS, Coltro PS, Farina Junior JA. Terapia por pressão negativa no tratamento de feridas complexas. Rev Col Bras Cir. 2017;44(1):81-93. DOI: $\underline{10.1590 / 0100-69912017001001}$

17 - Figueiredo TR, Pinheiro LLS, Correia PP, Valença MP, Bezerra SMMS. Tratamento de lesão por mediastinite com terapia assistida a vácuo. REDCPS 2014;1(1):31-6. DOI: $10.5935 / 2446-$ $\underline{5682.20150005}$

18 - Kamamoto F, Lima ALM, Rezende MR, MattarJunior R, Leonhardt MC, Kojima KE, et al. A new low-cost negative-pressure wound therapy versus a commercially available therapy device widely used to treat complex traumatic injuries: A prospective, randomized, non-inferiority trial. Clinics 2017;72(12):737-42. DOI: 10.6061/clinics/2017(12)04

\footnotetext{
Nota: Este artigo é produto da monografia de Carolina Letícia dos Santos Cruz no Curso de Especialização em Estomaterapia da Escola de Enfermagem da Universidade Federal de Minas Gerais.

Recebido em: 25/06/2020

Aprovado em: 08/10/2020
} 